\title{
QoS-based Semantic Micro Services Discovery and Composition using ACO Algorithm
}

\author{
Case Study: e-Learning Platform
}

\author{
Ahmed ESSAYAH ${ }^{1}$, Mohamed Youssfi ${ }^{2}$, Omar Bouattane $^{3}$, Khalifa Mansouri ${ }^{4}$, Elhocein Illoussamen $^{5}$ \\ Laboratory SSDIA, ENSET Mohammedia, Hassan II University of Casablanca \\ Mohammedia 28999, Morocco
}

\begin{abstract}
In this paper, we present a new model of e-Learning platforms based on semantic micro services using discovery, selection and composition methods to generate learning paths. In this model, each semantic micro service represents an elementary educational resource that can be a course, an exercise, a tutorial or an evaluation implementing a precise learning path objective. The semantic micro services are described using ontologies and deployed in multi-instances in a cloud environment according to a load balancing and a fault tolerance system. Learners' requests are sent to a proxy micro service having learning paths abstract structures represented as an oriented graph. Proxy micro service analyses the request to define the learner profile and context in order to provide him with the semantic micro services responsible of the educational resources satisfying his functional and non-functional needs. In this model, to achieve an optimal learning path generation a two steps process is employed, where local optimization uses semantic discovery and selection based on a matchmaking algorithm and a quality of service measurement, and global optimization adopts an ant colony optimization algorithm to select the best resource combination. Our experimental results show that the proposed model can effectively returns optimized learning paths considering individual, collective and pedagogical factors.
\end{abstract}

Keywords-Semantic micro service; quality of service; learning path; e-learning platform; service discovery and composition; ant colony optimization algorithm

\section{INTRODUCTION}

Information technologies have brought significant progress to companies' information systems. In the last few years, web technologies, virtualization architectures and cloud computing have contributed to the dimension development of information systems and have forced the companies to restructure to adapt to these new technologies and be in a position to innovate constantly in order to remain competitive. Mobile geolocalized systems like smart phones and tiny embedded systems as Raspberry and Arduino have opened a new era for universal objects interconnection.

The Web 3.0 is starting to take shape and Internet of Things (IoT) has become a reality that information systems have to take into consideration. In fact, our environment objects can embed processing units making them able to perform complex tasks, take decisions while permanently connected to the internet allowing them to take advantage of massive collective intelligence. The huge quantity of data (Big Data) produced by the connected objects and mobile applications, requires new data storage and processing technologies. In the computerassisted learning, the e-Learning is an educational mode that has to benefit from the conjunction of all these new information technologies and from all presential classes capabilities.

To tackle the massification of higher education, the automation of learning process has become crucial; it allows the autonomous and remote learning and the individualization of learning paths. Many research works have been interested in improving educational content presented in e-Learning platforms [1, 3, 10, 15]. Other researches have proposed approaches for learning paths optimization $[6,11]$. In another stage, some researchers have interested in learners behavior modeling in order to enhance the e-Learning platform performances $[23,25,26]$. In a rapidly changing and increasingly challenging environment, educational material description, discovery and composition require efficient strategies for better reuse. Micro services [8, 14, 20, 27] is an architecture style emerged in last few years that corresponds most closely to the massive distributed systems. The main idea is to break down a huge application to many tiny components loosely coupled and independent called "Micro Services". Each micro service should be responsible of one functionality, developed and deployed independently.

Different semantic languages allow describing functionalities of a micro service in a machine interpretable form using Semantic Web technologies [9, 22, 31, 16, 21]. The main purpose of semantic Web service technologies is to minimize the manual discovery and usage of Web services, by allowing software agents and applications to automatically discover, select, and invoke these Web resources to achieve the user needs. Many researches have interested in Semantic Web service discovery algorithms [5, 13, 24, 32] and proposed several matchmaking techniques to measure similarity score between user requirements and service capabilities.

Semantic micro service architecture seems to represent a great opportunity for learning management systems (LMS) [26, 7]. In this context, we propose a new model of e-Learning platform based on semantic micro services using semantic description, discovery, selection and composition for learning paths. In this model, each semantic micro service represents an elementary educational resource that can be a lesson, an exercise, a tutorial or an evaluation implementing a precise learning path purpose. Learners' requests are sent to a proxy micro service having learning paths abstract structures 
represented as an oriented graph. Proxy micro service analyses the request to define the learner profile and context in order to provide him with the semantic micro services responsible of the educational resources satisfying his functional and nonfunctional needs. In this model, to achieve an optimal learning path generation a two steps process is employed, where local optimization uses semantic discovery and selection based on a matchmaking algorithm and QoS measurement [28], and global optimization adopts an ant colony optimization algorithm [2, 11, 12, 33] to select the best resource combinations.

The remainder of this paper is organized as follows. First, some related work about learning path and e-Learning platforms personalization and optimization is discussed. In the second section we present our e-Learning platform model architecture considering a learning path presented by an oriented graph. We then describe different components making up our model. Next, we discuss QoS normalization and aggregation for various non-functional characteristics of micro services. This is followed by a description of the semantic micro service discovery and selection algorithm. We then apply the Ant Colony Optimization algorithm to the learning path in order to obtain the optimal solution for each learner according to his profile and context. An application of our model and results are discussed in the next section. Finally, we present some conclusions and future works.

\section{RELATED WORK}

Research efforts in learning paths generation and optimization in e-Learning platforms try to automate the learning process by generating personalized learning paths according to learners' profiles and preferences. The author in [35] proposes an adaptive learning path method that applies Ant Colony Optimization method and Ausubel Meaningful Learning Theory to find the best learning path for a group of learners according to their functional needs. The author in [30] uses a prediction method based on pattern recognition technique to identify dynamically the best learning style. In $[34,36]$ authors used artificial intelligent techniques to perform automatic personalization in e-learning systems. Bayesian networks are used in [37] to provide a solution of adaption of learning activities. In addition, service oriented architectures and ontologies are proposed in [38] to generate personalized learning resources. Most of existing approaches do not consider individual factors such as excellence, history, language, and motivation. In this paper, we present a model considering individual, collective and pedagogical factors in order to provide learners with the best learning experience.

\section{E-LEARNING PlATFORM MODEL ARCHITECTURE}

We consider a learning path represented by an oriented graph $\mathrm{G}\left(O_{i}, E_{j}\right)$ where nodes $O_{i}$ represent precise objectives, ( $i=1$ to $N$, where $N$ is the total number of objectives), and $E_{i}$ are edges representing all the possible paths a learner can use to complete the training goals, $(j=1$ to $M$, where $M$ is the total number of edges). For each node $i$, various implementations for educational resources can be proposed $R(i, k),(k=1$ to $H$, where $H$ is the number of resources associated to a node). There may be multiple teachers proposing relevant educational content for the same node $i$. The educational resource may be an entire course, a chapter, an exercise, a tutorial or an evaluation as a form of multiple choice or true/false quizzes. During learning process, a learner explore one educational resource for each node in the learning path, we use a local optimization method based on semantic matchmaking calculation and QoS measurements to select the right resources according to learner profile. Over the time, we use learners feedbacks and evaluations results to assign to each educational resource $R(i, k)$, of a node $i$ a score $S_{i}$ and to each edge $E_{j}$ a weight $W_{j}$ reflecting its pertinence. Our proposed platform use a metaheuristic optimization algorithm to choose the optimal learning path that provides the best results. Fig. 1 shows an example of a learning path composed by eight nodes and nine edges.

To reach an objective $O_{i}$ we define multiple possible implementations of an educational resource $R(i, k)$. In this model, we present each implementation of an educational resource as a semantic micro service $S M S-R_{i, k}$ described using ontologies and deployed in multi-instances in a cloud infrastructure. The motivation behind this choice is that each teacher may respond to an objective $O_{i}$ according to his pedagogical approach. All resources semantic micro services $S M S-R$ are registered in a special micro service called $S M S-D$ representing the platform semantic repository. $S M S-D$ uses an Ontology Web Language OWL-S [21] to describe properties and capabilities of $S M S-R$ in unambiguous and computerinterpretable form. In our model, we also define a special micro service called $S M S-C$, designed to centralize and expose configuration of all the platform' micro services. All learners requests are sent to a proxy micro service $S M S-P$ having learning paths abstract structures represented as an oriented graph. $S M S-P$ communicates with $S M S-D$ to define the learner profile and context and provide him with the $S M S-R$ responsible of the educational resources satisfying his functional and non-functional needs. In some cases, the proxy service needs to send an information to all or most of the platform micro services, in this particular case a highperformance communication system based in AMQP protocol is used by implementing one of its brokers such as Rabbit MQ. Fig. 2 shows the technical architecture of all micro services in our platform.

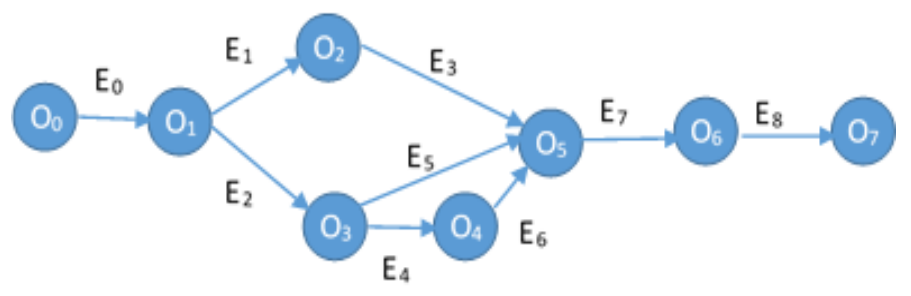

Fig. 1. Representation of a Learning Path as an Oriented Graph. 


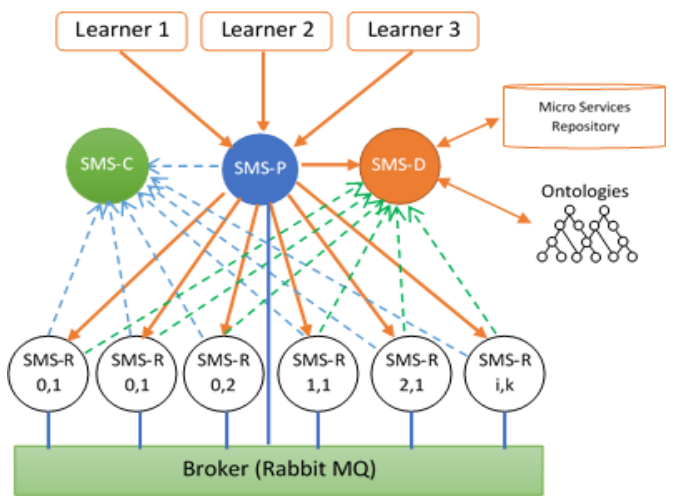

Fig. 2. e-Learning Platform Model Architecture.

\section{E-LEARNing PlatForm MODEL COMPONENTS DESCRIPTION}

\section{A. Educational Resource Semantic Micro Services SMS-R}

This semantic micro service is the main component of our e-Learning platform; it allows learners to access to educational resources. When an $S M S-R$ is started, it looks firstly for its configurations into to the configuration micro service $S M S-C$, then, it connects to the discovery micro service $S M S-D$ to register its location and publish its description to the semantic micro service registry. Technically, an educational resource micro service have three layers:

- Data access layer ensures educational content structuration and persistence in a lightweight data storage system such as micro DBMS or simple XML files. Generally, the educational resource content is an aggregate of a course presented as media content (text, images, audio, video, animation), an exercise, a tutorial and an evaluation.

- Business access layer defines the treatments required to manage the educational resource content. Operations in this layer guarantee the coherence of the data stored during interactions with the learner. It is the case of an initial assessment of a learner's skills for example.

- Web access layer allows exposing the business treatments via a REST API or through other remote access components such as web services based on SOAP protocol. Access rights of this layer are limited to the proxy micro service that manage learners' access to resources efficiently.

Educational resource semantic micro service evolves inside a container assuring the Inversion of Control (IoC) principle using an IoC micro framework. This framework manages implicitly the technical aspects of $S M S-R$ such as security, transactions management, persistency, logs, monitoring, migration in order to expose the micro service capabilities through HTTP or SOAP protocol, a web container is used. Fig. 3 shows an educational resource semantic micro service structure.

Educational resource semantic micro services functional and non-functional properties are described using an Ontology Web Language OWL-S [17, 18] in unambiguous and computer-interpretable form. An OWL-S description is composed of three parts, which are service profile, service model and service grounding. The service profile describes service functional and non-functional properties and it is the part used in the discovery process. The service model describes how the service works (internal processes), and the service grounding specifies the details of how the service can be accessed.

\section{B. Proxy Micro-Service SMS-P}

As mentioned in the previous section, we send all learners' requests to the proxy micro service, $S M S-P$ analyze the request and extract information about learner profile and context, learning path id and current objective id. $S M S-P$ send a discovery request with the objective description to the discovery micro service $S M S-D$ in order to get a sorted list of $S M S-R$ responsible of the educational resources matching exactly the current learning path objective and learner preferences. Using a matchmaking algorithm combined with a QoS measurement model, the proxy micro service selects the convenient $S M S-R$ and send it the learner request in order to get the educational resource. Based on learner feedback and evaluation results a pertinence score is assigned to the educational resource. This score will be used in addition to other parameters to calculate the weight of the edge linking the current resource with the previous nodes in the learning path. This process is a part of learning path optimization using a metaheuristic algorithm called Ant Colony Optimization Algorithm ACO. Our proposed optimization algorithm will be presented in Section 6, it proposes to the learner the optimal learning path from all paths defined in the training plan. Communication diagram in Fig. 4 shows the interactions between different platform actors.

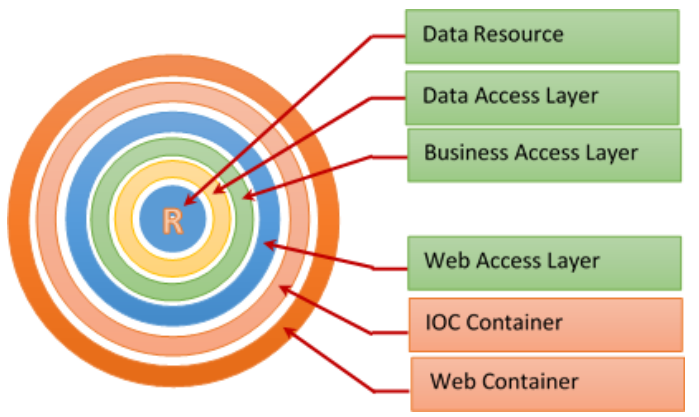

Fig. 3. Educational Resource Semantic Micro Service Structure.

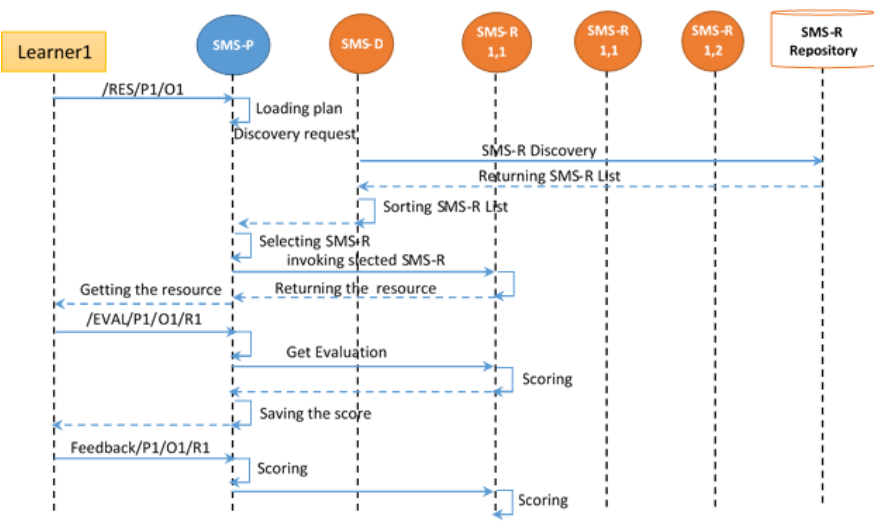

Fig. 4. Communication Diagram, Showing the Platform Actors' Interactions. 


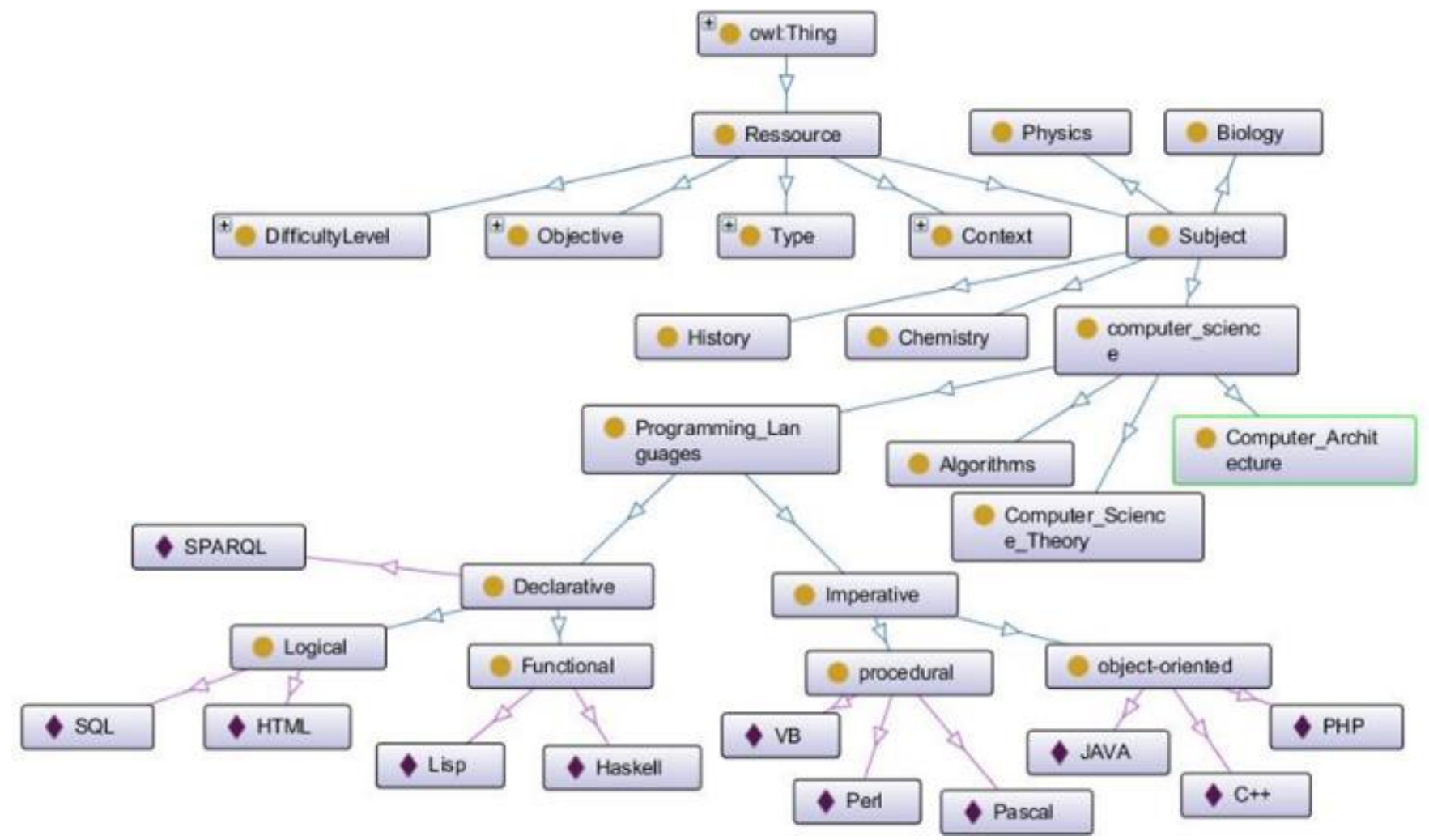

Fig. 5. Fragment from our Platform’ Ontology for Educational Resource.

\section{Semantic Discovery and Selection Micro Service SMS-D}

All $S M S-R$ are described using OWL-S in unambiguous and computer-interpretable form allowing semantic discovery and selection micro service $S M S-D$ first to compute semantic matchmaking of $S M S-R$ capabilities (which are expressed in service profile in terms of inputs and outputs) and the learning path objective description. Semantic matchmaking is performed by calculating the semantic distance between concepts that are defined in an ontology and used to describe $S M S-R$ and learning path nodes objectives. Matching degree and QoS attributes scores are used by $S M S-D$ to sort the discovered $S M S-R$ list before returning it to the proxy micro service. QoS of a $S M S-R$ refers to non-functional properties including availability, security, language, response time, price and reputation. Fig. 5 shows a fragment from our e-Learning platform ontology.

\section{Configuration Micro Service SMS-C}

In a micro service architecture, each micro service have its own configuration. This can result in duplication of a single property value across multiple micro services. Configuration micro service $S M S-C$ is designed to externalize, centralize and expose configuration of all micro services in one place. SMS-C allows us to save time and effort when one or multiple properties values need to be changed.

\section{QOS ATtRIBUTES NORMALIZATION}

QoS refers to non-functional properties of learning resources, including availability, security, language, response time, price and reputation. QoS attributes hold two different directions of their values; if the direction of the attribute is positive, it means that a bigger value is better. On the contrary, if the direction is referred as negative, it means that smaller values are better. For example, for attribute response time the smaller value is usually preferred, so the direction of this parameter is negative, whereas for attribute availability the bigger value indicates a better quality for the specified parameter, so the direction is positive. To address this issue, all QoS attributes need to be normalized to the same scale [28]. Generally, all QoS attributes value will be normalized into a range from 0 to 1 . The normalization is given by:

$q_{1}^{\prime}=\left\{\begin{array}{r}\frac{q-\min }{\max -\min }, \max \neq \min \\ 1, \max =\min \end{array}\right.$
$q_{2}^{\prime}=\left\{\begin{array}{r}\frac{\max -q}{\max -\min }, \max \neq \min \\ 1, \max =\min \end{array}\right.$

If the direction of attribute $q$ is positive, use $q_{1}^{\prime}$ for normalization, otherwise use $q_{2}^{\prime}$.

Each QoS attribute in Table I represents one aspect of quality of service, to evaluate the resource micro service QoS score we adopt an utility function given by formula 2 where $q_{i}$ represents the normalized value of $i^{\text {th }}$ QOS attribute and $w_{i}$ is the corresponding weight. By using weight $w_{i}$ for each QoS attribute, the system becomes able to calculate the resource QoS score according to each learner preferences. This method was proposed by [19], and is one of the widely used approaches in service selection and composition.

$Q o S(S M S-R)=\sum_{i=1}^{n} q_{i} w_{i}$ 
TABlE I. TyPICAL QoS ATtributes of A Resource Micro SERVICE

\begin{tabular}{|l|l|l|}
\hline QoS attribute & Description & Unit \\
\hline $\begin{array}{l}\text { Response time } \\
(\boldsymbol{R T})\end{array}$ & $\begin{array}{l}\text { the duration between request submission } \\
\text { and response reception }\end{array}$ & $\mathrm{ms}$ \\
\hline Availability $(\boldsymbol{A})$ & $\begin{array}{l}\text { the probability that a service is operating and } \\
\text { accessible }\end{array}$ & percent \\
\hline Price $(\boldsymbol{P})$ & $\begin{array}{l}\text { The money that a learner has to pay to use } \\
\text { the micro service }\end{array}$ & Euro \\
\hline Reputation $(\boldsymbol{R})$ & The quality of being trustworthy & percent \\
\hline
\end{tabular}

In our platform, we sort the candidate micro services according to their QoS score and semantic matching score, which reduces significantly the computational overhead of the overall optimization process.

A learning path is composed by multiple resources micro services to respond to a training pedagogical objective. Its QoS level for a precise learner is the addition of all QoS scores resource micro services composing it.

$Q o S(P)=\sum_{J=1}^{N} \operatorname{QoS}\left(S M S-R_{j}\right)$

While $N$ represents the total number of nodes for the learning path $P$ and $Q o S\left(S M S-R_{j}\right)$ is the quality of service sore for the node $j$.

\section{Semantic Micro SeRvice Discovery AND SELECTION}

Semantic micro service discovery is the process of locating existing Web services based on the semantic description of their functional and non-functional properties. In our platform, semantic micro service discovery is used to select the best candidate resource micro services $S M S-R$ satisfying a precis objective described semantically in the learning path. Fig. 6 shows how the discovery and selection process is performed.

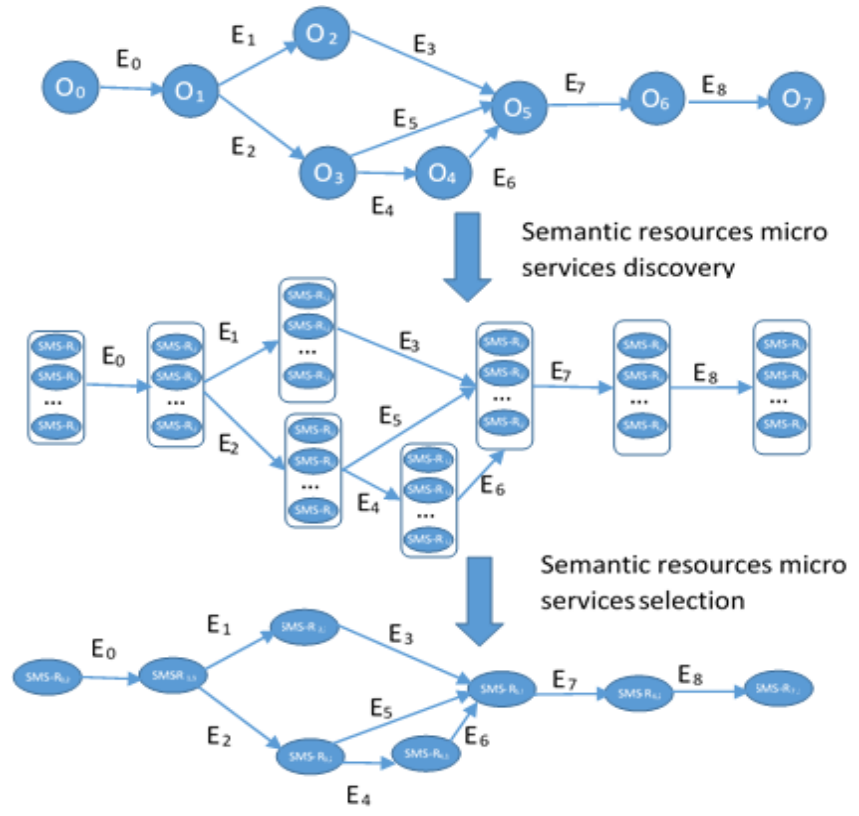

Fig. 6. Semantic Micro Service Discovery and Selection Process.
In this paper, we use our own domain ontology have been shown in Fig. 5 to calculate semantic distance $[4,29]$ between concepts composing SMS-R description and learning path objectives description. Our semantic matchmaking algorithm uses an edge-based approach to measure the semantic distance between two ontological concepts. In [18], the author computes the similarity between two concepts by finding the least common subsume $(L C S)$ node that connects their senses. For example, we can see in Fig. 5, the LCS of SQL and JAVA is Programming languages.

Once the LCS is identified, the distance between two senses is computed by:

$\operatorname{Sim}\left(c_{1}, c_{2}\right)=\frac{2 d}{L_{1}+L_{2}+2 d}$

Where $\mathrm{d}$ is the depth of the LCS from the root, $L_{l}$ is the path length between $c_{1}$ and $L C S$, and $L_{2}$ is the path length between $c_{2}$ and $L C S$.

Without considering the levels of nodes in the ontology hierarchy, the similarity measurement of this method could be misleading [32]. For example, the similarity between SQL and Java using this method is 0.57 and the similarity between Java and algorithms, which is a higher-level node, is 0.54 . We define a modified measure based on this method that takes into account the position of concept nodes in the ontology.

levelSim $\left(c_{1}, c_{2}\right)=\frac{2 d+S}{L_{1}+L_{2}+2 d+S}$

$S=\log \frac{2 * \max \left(L_{1}\right) * \max \left(L_{2}\right)}{\max \left(L_{1}\right)+\max \left(L_{2}\right)}$

Where $\mathrm{d}$ is the depth of the $L C S, L_{1}$ and $L_{2}$ are the path lengths between semantic node $c_{1}, c_{2}$ and the $L C S$ respectively, $\max \left(L_{1}\right)$ is the maximum path length of semantic node $c_{l}$, $\max \left(L_{2}\right)$ is the maximum path length of semantic node $c_{2}, S$ is the logarithm of the harmonic mean between $\max \left(L_{1}\right)$ and $\max \left(L_{2}\right)$. Formula (5) represents that the distance between any two nodes $c_{1}$ and $c_{2}$ is reduced if their parent node lies in the lower level of the hierarchy.

The similarity score between the micro service $S M S-R_{i}$ set of concepts $S C_{R}$ and a learning path' objective set of concepts $S C_{O}$ is computed as follows:

SimScore $\left(S C_{O}, S C_{R}\right)=\sum_{C_{O} \in S C_{O}} \frac{\text { levelSim }\left(c_{O}, S C_{R}\right)}{S C_{O}+S C_{R}}+$
$\sum_{C_{R} \in S C_{R}} \frac{\text { levelSim }\left(c_{R}, S C_{O}\right)}{S C_{O}+S C_{R}}$

\section{VII.LEARNING PATHS OPTIMIZATION USING ANT COLONY OPTIMIZATION ALGORITHM}

Ant colony optimization (ACO) algorithms [11] are bioinspired population-based metaheuristic algorithms modeling ants' behaviors. Ant system (AS) was the first ACO algorithm to be proposed in the literature by Marco Dorigo [2], in the 1920 s to find approximate solutions in a graph, the first algorithm was inspired by ants' behavior in searching a path between nest and food source. The original idea was diversified to tackle large-scale problems and several new algorithms have been created, inspired of multiple aspects of ants behavior. The first algorithm was originated of observation of food resources' exploitation by ants. In facts, an 
ant has a limited cognitive capability and by taking advantage of collective intelligence, it is able to find the shortest path between its nest and a food source.

The ants construct the solutions as follows:

- An ant explores randomly environment around the nest

- If this ant finds a food source, it returns directly to the nest, laying pheromone on its path.

- Pheromone is attractive; nearby ants tend to follow this path.

- Returning to the nest, these ants will increase pheromone in this path.

- If two paths are possible to reach the same food source, the shortest one will be followed, at the same time by more ants.

- The short path is increasingly promoted, becoming more and more attractive.

- The long path finished by disappearing because pheromone evaporates at certain rate in each iteration.

- Finally, all ants in the colony choose the shortest path.

In our case, we consider our learning path represented as an oriented graph in Fig. 1. Each node represents a precise objectives and each edge a possible navigation between two nodes. Each edge has a weight $W$ initialized firstly by pedagogical team to express a relative importance for different possible paths leaving the same node. Each learner exploring the graph represents an ant. While following a path $E_{j}$ to reach a node $O_{E}$ proposing knowledge evaluation, the ant lays pheromones $C$ which quantity depends on the score $\alpha$ obtained.

To reflect back-propagation phenomena, pheromones are not simply laid on the edge that leads the ant to the current node but on the $n$ previous edges that the ant has followed. This represents the pedagogical effect that the success of fail in a precise node is conditioned by learner prerequisites. Of course, this influence decreases with time and distance: more the node is far in the path history $P(a)$ of the ant $a$ less importance it has. To do, the quantity of pheromone lied decreases while the back-propagation moves backward. In our algorithm, we propose that the quantity $\alpha$ of pheromones that will be added to the edge $E_{k}$ in history $P(a)$ will be equal to $\alpha$ divided by $d(k)$, while $d(k)$ is the distance between the evaluation node $O_{E}$ and original node.

$C_{k}=C_{k}+\frac{\alpha}{d_{k}}$

Fig. 7 shows an example of back-propagation of pheromones for a learner passed an evaluation in node 7 and obtained a score $\alpha_{1}$. To reach the evaluation node $E_{7}$, he followed this learning path $P=\left\{O_{0}, O_{1}, O_{3}, O_{7}\right\}$. Quantities of pheromones laid on edges $E_{7}, E_{3}, E_{1}$, and $E_{0}$ will be respectively $\alpha_{1}, \alpha_{1 / 2}, \alpha_{1} / 3$ and $\alpha_{1 / 4}$.

The fact that pheromones evaporate biologically with time is extremely important, it allows the ant colony to update regularly the information used to define the optimal path. In our platform, it is important to implement an evaporation form to prevent our algorithm from being stuck in local optimum also to allow dynamic adaptability options.

Formula 9 shows the evaporation form of pheromones. $\tau$, the evaporation rate is a key feature in our system. The evaporation duration $x$ represents a range when the evaporation should be recalculated. Typically, $\tau=0.999$ and $x=1$ day.

$C_{t}=\tau^{x} C_{t-1}$

In addition to evaluation results, learners review and submit feedbacks about educational resources. Each educational resource get a score $\beta$ from learners. This score is multiplied by a credibility factor $\mu$ associated to each learner by the system and pedagogical team according to learner' attendance rate and honesty. Appreciation score $\beta$ is transformed to pheromone $A$ laid on previous edges that the learner has followed to reach the current resource while respecting the back-propagation principal as follows:

$A_{k}=A_{k}+\frac{\mu \beta}{d_{k}}$

Evaporation of pheromones $A$ is calculated the same way as formula 9 .

$A_{t}=\tau^{x} A_{t-1}$

Values of $W, C$ and $A$ are considered as collective factors, they are used by all learners to construct their own path. $W$ is an external factor given by pedagogical team to the learners/Ants community. $C$ and $A$ are internal factors created by individuals to serve the community. Our system should be able to consider individuals factors, related to learner profile and preferences, in order to achieve the desired compromise between individual, community and environment.

Individual factors that we may consider are numerous (preferences, excellence, history, language). The idea is to allow the system to propose solutions according to learner profile. In our case, we choose $H$ the history factor.

The history factor $H$ contains information about nodes visited previously with the learner, because it is an individual factor, there is an $H$ value per node for each learner. Default value of $H$ is 1 , when a node is visited, corresponding value of $H$ will be multiplied by a factor $h$ inversely proportional to the evaluation score. For example, we can choose these values:

- $\mathrm{h}=0.25$ if the score is between $75 \%$ and $100 \%$

- $\mathrm{h}=0.5$ if the evaluation score is between $50 \%$ and $75 \%$

- $\mathrm{h}=0.75$ if the evaluation score is between $25 \%$ and $50 \%$

- $\mathrm{h}=0.95$ if the evaluation score is less than $25 \%$

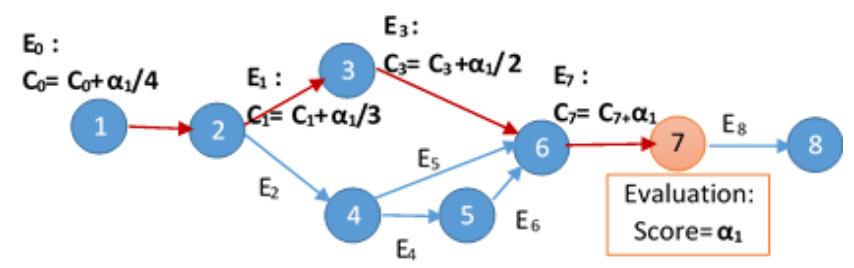

Fig. 7. Pheromones Back-Propagation Principal. 
The role of $H$ is to decrease the probability that a visited node will be proposed again. Of course, if a node gets a bad evaluation score $(h=0.95)$ it will be proposed very fast than if it gets a high score $(\mathrm{h}=0.25)$.

With time, learners forget what they had seen. It is for this reason that $H$ tends to return naturally to one. This antievaporation of pheromones phenomena is described as follows:

$H_{t}=H_{t-1}\left(1+\frac{1-H_{t-1}}{H_{t-1}} \frac{1-e^{\gamma x}}{1+e^{\gamma x}}\right)$

$\gamma$ is a constant of time that sets the anti-evaporation speed, it should be adjusted to correspond to learner' memory volatility. It is a pedagogical constant, which should be the subject of a discussion with teachers. Formulas 12 and 13 allow an easy calibration of $\gamma$. Formula 14 have shown how to compute $\gamma$ value using $H$ in the moments $t$ and $t-1$ and the period $x$ during which a learner may eventually forget a viewed resource.

$\gamma=\frac{1}{x} \ln \left(\frac{1+\tau}{1-\tau}\right)$

$\tau=\frac{H_{t}-H_{t-1}}{1-H_{t-1}}$

All factors described previously $W, C, A$ and $H$ are unified in a form of a function called "Fitness", by analogy with literature of genetics algorithms. Formula 15 shows the expression of the fitness value of edge $E_{i, j}$ linking node $i$ with node $j$ for a learner $a$. $H$ is the memory factor, $W$ is the pedagogical weight, $C$ is evaluation score pheromones and $A$ is the feedback score pheromones. $\omega_{H}, \omega_{w}, \omega_{c}$ et $\omega_{A}$ are weights allowing giving more emphasis to a factor compared to others. This function calculates excellence and desirability of an edge, and privileges it in the selection process.

$f\left(a, E_{i, j}\right)=\omega_{H} H(j, a)\left(\omega_{w} W+\omega_{c} C+\omega_{A} A\right)$

We can say that an edge is desirable if:

- The pedagogical team reinforces it (high $W$ ).

- It is a source of good evaluation scores (high $C$ )

- It has a good feedback score (high $A$ )

- The next node had never been visited by the learner or estimated forgotten ( $H$ near to 1$)$

When a learner validates a node, he needs to choose between the available edges the suitable one. It is in this level where fitness measurements are used. A selection process chooses an edge randomly but most likely, it has a high fitness value. Thus, it appears clearly that effective edges become dominating but not exclusively. There is always place of chance and exploration. This chance is a crucial characteristic of selection process we talk here about "selective pressure" $s$. More $s$ has a big value, more the fitness value is considered in selection process and strong edges tend to dominate weak ones. There are various methods to create the selection algorithm and consequently setup the parameter $s$. In our platform, the used common methods are:
- Roulette-wheel selection strategy-the probability to select each edge is strictly proportional to its fitness value; this strategy is entirely automatic, there is no way to setup the selective pressure. Roulette-wheel selection ensures that better edges have higher chance to be chosen and weak edges will be disappeared, and exploration will be replaced by a leaded exploitation. Formula 16 expresses the probability of selection of an edge $E_{i, j}$ linking node $\mathrm{i}$ by node $\mathrm{j}$ using roulette-wheel strategy.

$p\left(a, E_{i, j}\right)=\frac{f\left(a, E_{i, j}\right)}{\sum_{k \epsilon E} f\left(k, E_{k, j}\right)}$

- Tournament selection: It is a method of selecting randomly $s$ individuals from a population and the winner of each tournament (the one with the best fitness) is selected. We can see clearly that $s$ influences selection pressure. If the tournament size is larger, weak individuals have a smaller chance to be selected, because, if a weak individual is selected to be in a tournament, there is a higher probability that a stronger individual is also in that tournament. This method has the advantage of being easily configurable.

- Stochastic tournament selection: In this method, the weaker edge is selected by default. $s_{1}$ challengers are selected randomly from a population, successive edges are compared and the edge with higher fitness value is inserted in the new population. The process continues until the population is full.

\section{APPLICATION AND MODEL EVALUATION}

In this section, we discuss experimental results corresponding to an application implemented in the proxy micro service that lunches randomly HTTP clients representing each a learner. Each learner follows his learning path navigating from a node to another in the learning path graph (Fig. 8). We have chosen as an example a portion of a learning path about learning oriented-object programming OOP with Java language, having the following structure.

This learning path is an oriented graph composed by the following nodes:

- Nodes 1, 2, 5, 9 and 13 represent courses.

- Nodes 3, 6, 10 and 14 represent exercises.

- Nodes 4, 7,11 and 15 represent tutorials.

- Nodes 5, 8, 12, 16 and 17 are evaluations.

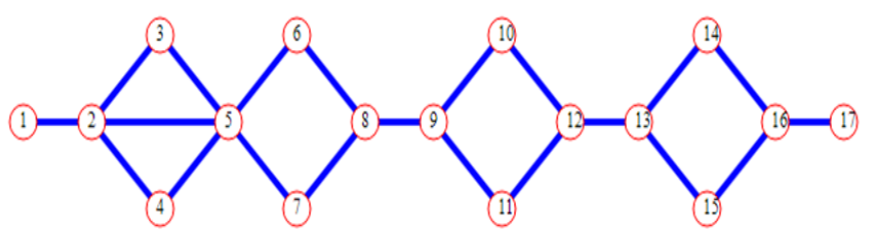

Fig. 8. The Structure of Proposed Learning Path. 
Static data of this pedagogical structure has been implemented in an XML file, which the structure is shown in Fig. 9.

In this simulation, we consider that in node 2 , that is a lesson, discovery algorithm found four semantic micro services $S M S-R$ matching exactly the objective description but with different QoS values. Table II shows different qualities of service values discovered in node 2 .

Three learners expressed their QoS preferences in Table III, allowing the system to rank selected resources micro services according to learners' profiles.

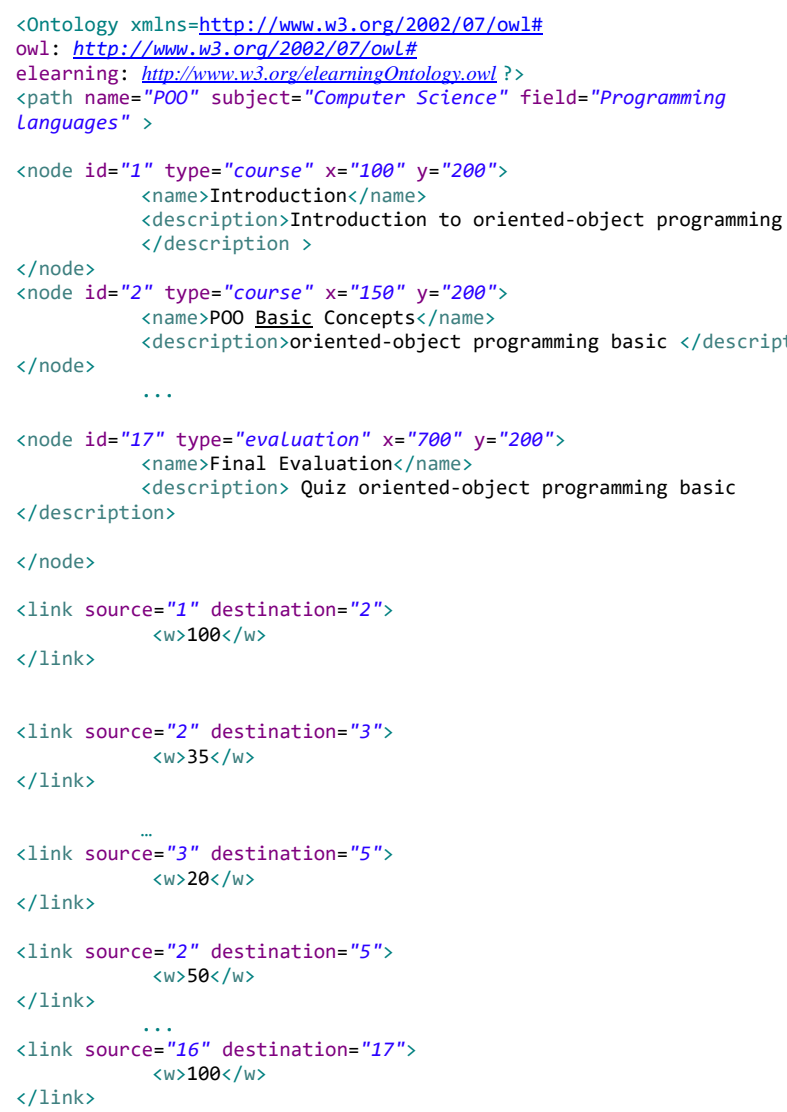

Fig. 9. XML File Represanting the Application Learning Path Structure.

TABLE II. QOS VALUES OF SMS-R IN NODE 2

\begin{tabular}{|c|c|c|c|c|}
\hline & $S M S-R_{2,1}$ & $S M S-R_{2,2}$ & $S M S-R_{2,3}$ & $S M S-R_{2,4}$ \\
\hline Response time & $3 \mathrm{~ms}$ & $10 \mathrm{~ms}$ & $5 \mathrm{~ms}$ & $20 \mathrm{~ms}$ \\
\hline Availability & $90 \%$ & $70 \%$ & $98 \%$ & $80 \%$ \\
\hline Price & 15 Euros & 9 Euros & 50 Euros & 29 Euros \\
\hline Reputation & $60 \%$ & $40 \%$ & $80 \%$ & $50 \%$ \\
\hline
\end{tabular}

TABLE III. LEARNERS QOS PREFERENCES

\begin{tabular}{|l|l|l|l|}
\hline & Learner $_{1}$ & Learner $_{2}$ & Learner $_{3}$ \\
\hline Response time & 0.2 & 0.1 & 0.4 \\
\hline Availability & 0.1 & 0.6 & 0.1 \\
\hline Price & 0.3 & 0 & 0.4 \\
\hline Reputation & 0.4 & 0.3 & 0.1 \\
\hline
\end{tabular}

TABLE IV. QOS SCORES ACCORDING TO LEARNERS PREFERENCES

\begin{tabular}{|c|c|c|c|c|}
\hline & $S M S-R_{2,1}$ & $S M S-R_{2,2}$ & $S M S-R_{2,3}$ & $S M S-R_{2,4}$ \\
\hline Learner $_{1}$ & 0.72 & 0.41 & 0.67 & 0.28 \\
\hline Learner $_{2}$ & 0.67 & 0.05 & 0.88 & 0.28 \\
\hline Learner $_{3}$ & 0.86 & 0.63 & 0.55 & 0.26 \\
\hline
\end{tabular}

We notice that invoked resource in node 2 change according to learners' preferences (Table IV). For learner ${ }_{1}$ and learner $_{3}$ who are interested more about service price and response time the resource micro service $S M S-R_{2,1}$ with QoS scores equal to 0.72 and 0.86 is the best match. The $S M S-R_{2,3}$ with QoS score equal to 0.88 is the most suited to learner2 who has more interest in the micro service availability and reputation.

In this simulation, scoring system is on 100 . When multiple edges leave the same node, we choose pedagogical pertinence values $W$ allowing the recommendation of tutorials instead of exercises, it is the case in edges $(2=>3),(2=>4)$ and $(2 \Rightarrow>5)$ having respectively the values $W: 35,45$ and 20 . Which means that the pedagogical team recommends to follow the course by the tutorial $4(45 \%)$ or the exercise $3(35 \%)$ or as a last choice the next course $5(20 \%)$.

By visiting a knowledge evaluation node, the ant lays pheromones $C$ which quantity depends on the result obtained by the learner (Formula 7). In addition, a feedback score representing the quantity of pheromones $A$ (Formula 9) corresponding to the learner feedback. Before moving to the next node, we activate the evaporation process (Formula 8 and 10).

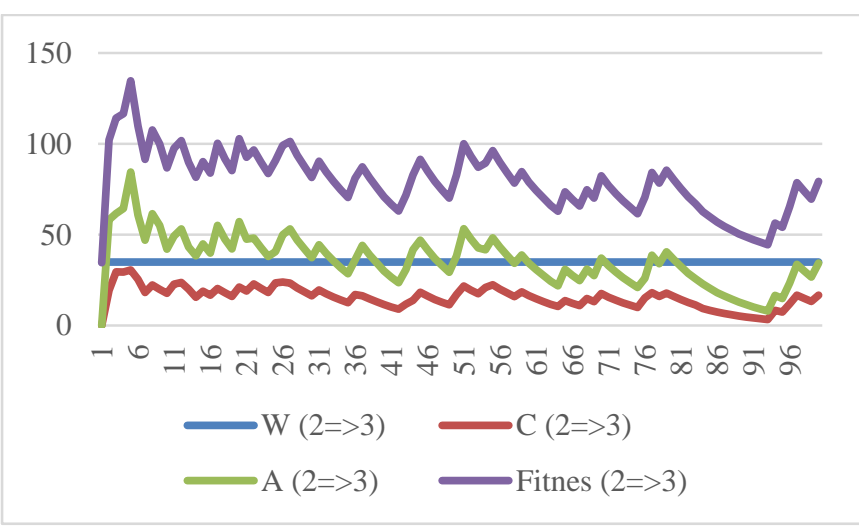

Fig. 10. Evolution of $W, C, A$ and Fitness Values for the Edge $2=>3$.



Fig. 11. Evolution of $W, C, A$ and Fitness Values for the Edge $2=>4$. 


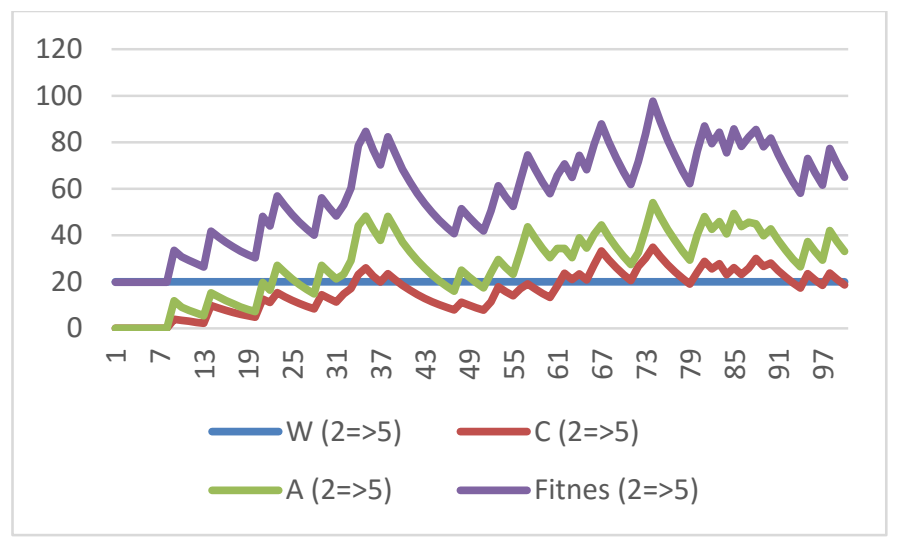

Fig. 12. Evolution of $W, C, A$ and Fitness Values for the Edge $2=>5$.



Fig. 13. Fitness Values Comparison for Edges $2=>3,2=>4$ and $2=>5$.

To calculate the fitness value for each edge, $C$ and $A$ pheromones quantities are normalized with the $W$ quantity putting them at 100 .

Fig. 10, 11 and 12, respectively shows evolution curves of parameters $W, C, A$ and Fitness while learners are following this learning path.

Fig. 13 represents a comparison between fitness values of three edges $2=>3,2=>4$ and $2=>5$. We notice that the edge $(2=>4)$ is the most followed, which reinforces the pedagogical team recommendation. Since the system uses many random selection methods, domination can changes with time. It is the case for learner number 57 who makes the edge $(2=>3)$ temporary a dominating one. It is the case also for learner 73 who followed the edge $(2=>5)$ making it temporary dominating instead of the recommended one. This allows new parameters difficult to take into consideration to appear such as excellence, learners serenity and evaluations pertinence. Despite all of that, our model allows to get at the end, very interesting results resisting all mentioned aleatory phenomena.

\section{CONCLUSION}

In this paper, we presented a new model for e-Learning platform based on semantic micro services and using discovery, selection and composition methods to create high quality learning paths responding to learners' preferences.

In this model, to achieve an optimal learning path selection a two steps process are employed, where local optimization uses semantic discovery and selection based on a matchmaking algorithm and QoS measurement, and global optimization adopts an ant colony optimization algorithm to select the best resources combination. We consider individual, collective and pedagogical factors in order to provide learners with the best learning experience.

The achieved results are promising but there are still many potential extensions, which can enhance the platform reliability and performance. We are planning to improve the model by adding new advanced factors to the global optimization algorithm, and to enrich the resources micro services with additional semantic description approaches in order to improve the semantic matchmaking algorithms results.

\section{REFERENCES}

[1] Albano, G., M. Gaeta \& S. Salerno (2006) E-learning: a model and process proposal. International Journal of Knowledge and Learning, 2, 73-88.

[2] Colorni, A., M. Dorigo \& V. Maniezzo (1992) DISTRIBUTED OPTIMIZATION BY ANT COLONIES. Toward a Practice of Autonomous Systems: Proceedings of the First European Conference on Artificial Life, 134-142.

[3] Comendador, B. E. V., L. W. Rabago, B. T. Tanguilig \& Ieee (2016) An Educational Model Based on Knowledge Discovery in Databases (KDD) to Predict Learner's Behavior Using Classification Techniques. 2016 Ieee International Conference on Signal Processing, Communications and Computing (Icspcc), 6.

[4] Cui, J. \& H. Yasuhara (1995) Calculating word similarity based on common attributes, total semantic distance and concept frequency. Symbiosis of Human and Artifact: Future Computing and Design for Human-Computer Interaction, 20, 729-734.

[5] Gmati, F. E., N. Y. Ayadi, A. Bahri, S. Chakhar \& A. Ishizaka (2016) A Framework for Parameterized Semantic Matchmaking and Ranking of Web Services. Proceedings of the 12th International Conference on Web Information Systems and Technologies, Vol 1 (Webist), 54-65.

[6] Govindarajan, K., V. S. Kumar \& Kinshuk. 2016. Dynamic Learning Path Prediction - A Learning Analytics Solution. In IEEE 8th International Conference on Technology for Education (T4E), 188-193. Indian Inst Technol Bombay, Mumbai, INDIA: Ieee.

[7] Hegde, V. \& H. S. S. Rao. 2017. A Framework to Analyze Performance of Student's in Programming Language Using Educational Data Mining. In 8th IEEE International Conference on Computational Intelligence and Computing Research (IEEE ICCIC), 632-635. Tamilnadu Coll Engn, Coimbatore, INDIA.

[8] Jaramillo, D., D. V. Nguyen, R. Smart \& Ieee. 2016. Leveraging microservices architecture by using Docker technology. In SoutheastCon. Norfolk, VA.

[9] Jensen, J. (2019) A systematic literature review of the use of Semantic Web technologies in formal education. British Journal of Educational Technology, 50, 505-517.

[10] Juhanak, L., J. Zounek \& L. Rohlikova (2019) Using process mining to analyze students' quiz-taking behavior patterns in a learning management system. Computers in Human Behavior, 92, 496-506.

[11] Kamsa, I., R. Elouahbi, F. El Khoukhi, T. Karite, H. Zouiten \& Ieee. 2016. Optimizing collaborative learning path by ant's optimization technique in e-learning system. In 15th International Conference on Information Technology Based Higher Education and Training (ITHET). Istanbul, TURKEY: Ieee.

[12] Klasnja-Milicevic, A., B. Vesin, M. Ivanovic \& Z. Budimac (2011) ELearning personalization based on hybrid recommendation strategy and learning style identification. Computers \& Education,56, 885-899.

[13] Kumar, V. (2018) Selecting an Appropriate Web-Scale Discovery Service: A Study of the Big 4's. Desidoc Journal of Library \& Information Technology, 38, 396-402.

[14] Malavalli, D. \& S. Sathappan. 2015. Scalable Microservice Based Architecture For Enabling DMTF Profiles. In 11th International 
Conference on Network and Service Management (CNSM), 428-432. Barcelona, SPAIN.

[15] Miranda, S. \& P. Ritrovato (2014) Automatic extraction of metadata from learning objects. 2014 International Conference on Intelligent Networking and Collaborative Systems (Incos), 704-709.

[16] Moussallem, D., M. Wauer \& A. C. N. Ngomo (2018) Machine Translation using Semantic Web Technologies: A Survey. Journal of Web Semantics, 51, 1-19.

[17] Pan, S. L., Y. X. Zhang \& Ieee (2009) Ranked Web Service Matching for Service Description Using OWL-S. Wism: 2009 International Conference on Web Information Systems and Mining, Proceedings, 427431.

[18] Paolucci, M., T. Kawamura, T. R. Payne \& K. Sycara (2002a) Semantic matching of Web services capabilities. Semantic Web - Iswc 2002, 2342, 333-347.

[19] Paulraj, D., S. Swamynathan \& M. Madhaiyan (2011) PROCESS MODEL ONTOLOGY-BASED MATCHMAKING OF SEMANTIC WEB SERVICES. International Journal of Cooperative Information Systems, 20, 357-370.

[20] Peng, S. J., Z. T. Zhang \& C. L. Wu. 2016. Geological Cloud Platform Based on Micro Service Architecture. In Proceedings of the 3rd International Conference on Wireless Communication and Sensor Networks, eds. J. Wang, H. Wang, B. Cheng \& T. Chen, 566-568.

[21] Rozsa, V., A. F. G. Viera \& M. Dutra (2019) Semantic Web Technologies Applied to Internet Search Engines. Investigacion Bibliotecologica, 33, 165-191.

[22] Shafin, S. H., L. Zhang \& X. Xu. 2012. Automated Testing of Web Services System Based on OWL-S.

[23] Singh, H. \& S. J. Miah (2019) Design of a mobile-based learning management system for incorporating employment demands: Case context of an Australian University. Education and Information Technologies, 24, 995-1014.

[24] Somasundaram, T. S., R. A. Balachandar, V. Swaminathan, A. Kumar, V. Paramasivan \& Ieee. 2007. Semantic description and discovery of grid services using WSDL-S and QoS based matchmaking algorithm.

[25] Tseng, J. C. R., H. C. Chu, G. J. Hwang \& C. C. Tsai (2008) Development of an adaptive learning system with two sources of personalization information. Computers \& Education, 51, 776-786.

[26] Turker, Y. A., K. Baynal \& T. Turker (2019) The Evaluation Of Learning Management Systems By Using Fuzzy Ahp, Fuzzy Topsis And An Integrated Method: A Case Study. Turkish Online Journal of Distance Education, 20, 195-218.
[27] Wang, B., S. L. Yang, X. L. Ren, G. Y. Wang \& M. Assoc Comp (2017) Research on Digital Publishing Application System Based on MicroService Architecture. Proceedings of 2017 Vi International Conference on Network, Communication and Computing (Icncc 2017), 140-144.

[28] Wang, H. B., D. R. Yang, Q. Yu \& Y. Tao (2018) Integrating modified cuckoo algorithm and creditability evaluation for QoS-aware service composition. Knowledge-Based Systems, 140, 64-81.

[29] Wei, T. T. \& H. Y. Chang (2015) Measuring Word Semantic Relatedness Using WordNet-Based Approach. Journal of Computers, 10, 252-259.

[30] Yang, J., Z. X. Huang, Y. X. Gao \& H. T. Liu (2014) Dynamic Learning Style Prediction Method Based on a Pattern Recognition Technique. Ieee Transactions on Learning Technologies, 7, 165-177.

[31] Zhang, L. \& Ieee (2014) OWL-S Based Web Service Discovery in Distributed System. 2014 Ieee Workshop on Electronics, Computer and Applications, 882-885.

[32] Zhang, N., J. Wang, Y. T. Ma, K. Q. He, Z. Li \& X. Q. Liu (2018) Web service discovery based on goal-oriented query expansion. Journal of Systems and Software, 142, 73-91.

[33] Zhao, Q., Y. Q. Zhang, J. Chen \& Ieee (2016) An Improved Ant Colony Optimization Algorithm for Recommendation of Micro-learning Path. 2016 Ieee International Conference on Computer and Information Technology (Cit), 190-196.

[34] Markowska-Kaczmar U, Kwasnicka H. , Paradowski M. Intelligent Techniques in Personalization of Learning in e- Learning Systems. Studies in Computational Intelligence, Computational Intelligence for Technology Enhanced Learning, Vol. 273, 2010.

[35] Brusilovsky P. , Peylo C. Adaptive and intelligent Web-based educational systems. In P. Brusilovsky and C. Peylo (eds. ), International Journal of Artificial Intelligence in Education 13 (2-4), Special Issue on Adaptive and Intelligent Web-based Educational Systems, pp. 159-172, 2007.

[36] Ahmad, K., Maryam, B. I., \& Molood, A. E. (2013). A novel adaptive learning path method. 4th International Conference on e-Learning and eTeaching (ICELET 2013), 2013.

[37] Almohammadi, Khalid; Hagras, Hani; Alghazzawi, Daniyal; et al. A zSlices-based general type-2 fuzzy logic system for users-centric adaptive learning in large-scale e-learning platforms. SOFT COMPUTING Volume: 21 Issue: 22 Pages: 6859-6880, 2017.

[38] Zaharescu, EZaharescu, GA; Semantic Web Technologies Integrated in a SOA-Based E-Learning System, proceedings of the 6th international conference on virtual learning, icvl, 2011 